\title{
Rehabilitation of Edentulous Arches with Fixed Prosthesis Supported by Tilted Implants: A Systematic Review
}

\author{
Sudhindra Sudhakar Mahoorkar ${ }^{1}$, Somwanshi Adesh V², Manju George ${ }^{3}$
}

\begin{abstract}
Aim and objective: The aim of this systematic review was to seek evidence and to find the favorable and unfavorable factors for the use of a minimum number of implants in complete arch rehabilitation.

Materials and methods: A search of electronic database limited to English language articles was conducted using the following MeSH terms; "dental implant", "tilted implant", "axial implant", "edentulous patient", "edentulous maxilla", and "edentulous mandible", "implant supported dental prosthesis", "immediate loading and immediate placement". They were used alone or in combination. Thirty-three articles were selected for the final review which were all clinical studies with human participants and had a follow-up period of 1 year or more. In all the studies included in this review, a minimum of four implants were placed in each arch and one implant placed in each arch was angulated.

Results: Of the 33 articles analyzed, 21 (63.63\%) articles reported failure of implants. A total of 161 (1.56\%) implants failed out of the 10,300 implants placed. In the 161 failed implants, $63(39.13 \%)$ were axially placed implants and $63(39.13 \%)$ were implants place at an angulation. The orientation of $60(37.27 \%)$ implants was not mentioned.

Conclusion: The survival rate of tilted implants at the implant and prosthetic levels is good. Tilted implants provide greater surface area for osseointegration, provide greater primary stability, reduce cantilever length, reduce bone resorption, and also reduce the need for bone grafting. The angulation of the implant which provided the most success was found to be $30^{\circ}$.

Keywords: Axial implant, Fixed implant prosthesis, Tilted implant.

International Journal of Prosthodontics and Restorative Dentistry (2021): 10.5005/jp-journals-10019-1299
\end{abstract}

\section{INTRODUCTION}

The life expectancy of humans has increased in the last few decades due to the improved and continuous progress in the medical field. The loss of teeth due to old age and its replacement is seen as a natural phenomenon. The complete loss of teeth during the fourth and fifth decades of life due to various lifestyle habits and diseases is more common today. The increase in the level of education and improved patient awareness has led to an increase in patients demanding higher quality treatment options. Age-related tooth loss, anatomic condition of edentulous ridges, psychological needs, decreased performance of removable prostheses, and predictable long-term results of implantsupported prostheses have increased the demand for implantsupported rehabilitation of teeth.

Traditional treatment plans typically called for a large number of implants placed in fairly vertical positions throughout the entire arch. ${ }^{1}$ However, the rehabilitation of edentulous jaws with implants is often complicated by poor bone quality, especially in the posterior region, and reduced bone volume due to a long-term edentulous state. ${ }^{2}$ Alveolar bone resorption and pneumatization of the maxillary sinus reduce, in many cases, the available amount of bone in both width and height for the placement of dental implants in the edentulous posterior maxilla. ${ }^{3}$ In the mandible, the inferior alveolar nerve and associated structures may provide minimal bone for implant anchorage or prevent the placement of implants distal to the mental foramina altogether. ${ }^{1}$ Bone grafting procedures to increase the bone volume available for implant placement is a viable treatment option but they often require demanding surgical procedures and can lead to complications, morbidity, and high costs. Therefore, patient compliance is often poor. ${ }^{2}$ If posterior implants could not be placed and to compensate for 1,30partment of Prosthodontics, Crown and Bridge, HKES's SN Institute of Dental Sciences and Research, Kalaburagi, Karnataka, India ${ }^{2}$ Maharashtra Institute of Dental Science and Research Centre, Latur, Maharashtra, India

Corresponding Author: Manju George, Department of Prosthodontics, Crown and Bridge, HKES's SN Institute of Dental Sciences and Research, Kalaburagi, Karnataka, India, Phone: +91 9742899592, e-mail: manjuglams@gmail.com

How to cite this article: Mahoorkar SS, Adesh VS, George M. Rehabilitation of Edentulous Arches with Fixed Prosthesis Supported by Tilted Implants: A Systematic Review. Int J Prosthodont Restor Dent 2021;11(1):33-53.

Source of support: Nil

Conflict of interest: None

these biologic limitations, a lengthy cantilever distal to the terminal implant was typically needed to provide patients with adequate posterior dentitions; however, extensive posterior cantilevers are biomechanically unfavorable due to increased occlusal forces. ${ }^{1}$

To overcome such limitations, different therapeutic alternatives have been proposed, such as long distal cantilever (Shackleton et al.), ${ }^{4,5}$ short implants (Goené et al.; ${ }^{6}$ Renouard and Nisand; ${ }^{7}$ Maló et al. ${ }^{8}$ ) or implants placed in specific anatomical areas like, for the maxilla, the pterygoid region, the tuberosity of the zygoma (Khayat and Nader; ${ }^{9}$ Venturelli; ${ }^{10}$ Balshi et al. ${ }^{11}$ Brånemark et al.; ${ }^{12}$ Galán Gil et al.; ${ }^{13}$ Aparicio et al.;4 Maló et al. $\left.{ }^{15}\right)$. Any of these procedures requires considerable surgical expertise and has its advantages, limitations, surgical risks, and complications involving biological and financial costs. ${ }^{2}$ 
In recent years, several clinical studies have reported that placement of implants at an angulation is a feasible option (Krekmanov; ${ }^{16}$ Krekmanov et al. ${ }^{17}$ Aparicio et al. ${ }^{18}$ Maló et al. ${ }^{19,20}$ Calandriello and Tomatis; ${ }^{21}$ Capelli et al..;2 Agliardi et al. $\left.{ }^{23,24}\right){ }^{2}$ The introduction of tilted implants has provided a significant alternative for the restoration of maxillary and mandibular posterior segments without bone grafting. ${ }^{1}$ Tilted implants provide several surgical and prosthetic advantages, like the possibility of placing long implants with an improvement of bone anchorage, the reduction of the need for bone grafting, the avoidance of long cantilevers, and the possibility of increasing the distance between anterior and posterior abutments, with an improvement of the load distribution. ${ }^{2}$

The All-on- $4^{\oplus}$ treatment concept was introduced by Nobel Biocare AB, Göteborg, Sweden. This protocol using only four implants has produced good short-term outcomes, with a survival rate of $98.2 \%$ and marginal bone level of $0.6 \mathrm{~mm}$ at 6 -month follow-up. Since this first report, several other authors have reported good short- and medium-term outcomes for patients undergoing this treatment. Moreover, recent systematic reviews have confirmed these results for maxilla and mandible rehabilitation. ${ }^{24}$

The All-on- 6 treatment protocol is used to minimize the length of the cantilever. It is a deviation from the All-on-4 treatment modality.

The different treatment modalities provide various advantages to the clinicians as well as to the patients. But they also come with a variety of disadvantages too. The failure rate of such treatment modalities even though is less cannot be ignored. For this treatment option to be widely advocated and used successfully, one has to sort out the factors which favor and those which are unfavorable to this treatment option. The factors which contribute to the failure of such cases cannot be assessed from the reports of a handful of patients treated by a single clinician. Therefore, one needs to extensively study and carefully assess a considerable number of cases treated.

Thus, this review aims to seek evidence and to find the factors which are favorable and unfavorable for the use of a minimal number of implants for the complete arch rehabilitation of a patient.

\section{Materials and Methods}

An electronic search was carried out in PubMed and Medline. The keywords used for the search were "dental implant", "tilted implant", "axial implant", "edentulous patient", "edentulous maxilla", and "edentulous mandible", "implant-supported dental prosthesis", "immediate loading and immediate placement". They were used alone or in combination.

\section{Inclusion Criteria}

- The articles from 2005 to 2016 were included in the study.

- The articles selected were limited to in vivo studies involving human subjects with a minimum of 10 patients treated.

- Studies with a minimum of 4 implants and a maximum of 10 implants placed on a single arch of which at least two are tilted implants were considered.

- Articles with a minimum of 1-year follow-up and loss of study participants $<10 \%$ were included.

- Use of tilted implants.

- The survival rate of tilted and upright implants should be indicated and calculable from the data provided.

\section{Exclusion Criteria}

Multiple publications with the same pool of patients, single case reports, studies with missing data, and studies in languages other than English were excluded. Publications that did not meet the above inclusion criteria and those that were not dealing with original clinical cases (reviews and technical reports) were also excluded.

Studies dealing with orthodontic implants, mini-implants, partial rehabilitation, and removable prosthesis were excluded too.

Thirty-three articles were selected for the final review which were all clinical studies with human participants and had a follow-up period of 1 year or more. In all the studies included in this review, a minimum of four implants were placed in each arch and one implant placed in each arch was angulated. The restoration of an edentulous maxilla or mandible or both with implant retained fixed prosthesis is the best treatment option available currently with long-term patient satisfaction and comfort compared to other methods of teeth replacement.

\section{Outcomes}

After analyzing the selected articles the following outcomes were thoroughly analyzed.

- Type of study.

- Sample size.

- Number of implants placed.

- Number of implants placed in each arch.

- Angulation of the implant placed.

- The use of surgical guides for implant placement.

- The time of implant placement.

- The loading protocol was followed.

- The length of the cantilever.

- Type of provisional restoration/prosthesis.

- Type of opposing dentition.

- Follow-up interval.

- Follow-up period.

- Bone loss.

- Patient satisfaction.

\section{Results}

A total of 75 articles were obtained through the initial screening process. Out of which 28 articles were discarded as they did not fulfill the inclusion criteria. A total of 47 articles were identified as potentially eligible articles through screening by titles and abstracts. The full-text articles were obtained and thoroughly evaluated. As a result, 33 articles fulfilled the inclusion criteria and were included in the systematic review (Table 1, Fig. 1). The distribution of the type of study was mentioned in Table 2.

The review analyzed 15 criteria from each article, which include the type of study, sample size, number of implants used, number of implants placed per arch, the angulation of the implants placed, the use of surgical guides, the surgical protocol followed, time of placement of a provisional restoration, the length and presence of cantilever, type of opposing dentition, follow-up interval, follow-up period, bone loss at the implant site, patient satisfaction and the implant failures.

Of the 33 articles analyzed, 21 (63.63\%) articles reported failure of implants. A total of $161(1.56 \%)$ implants failed out of the 10,300 implants placed. In the 161 failed implants, 63 (39.13\%) were axially placed implants and 63 (39.13\%) were implants placed 


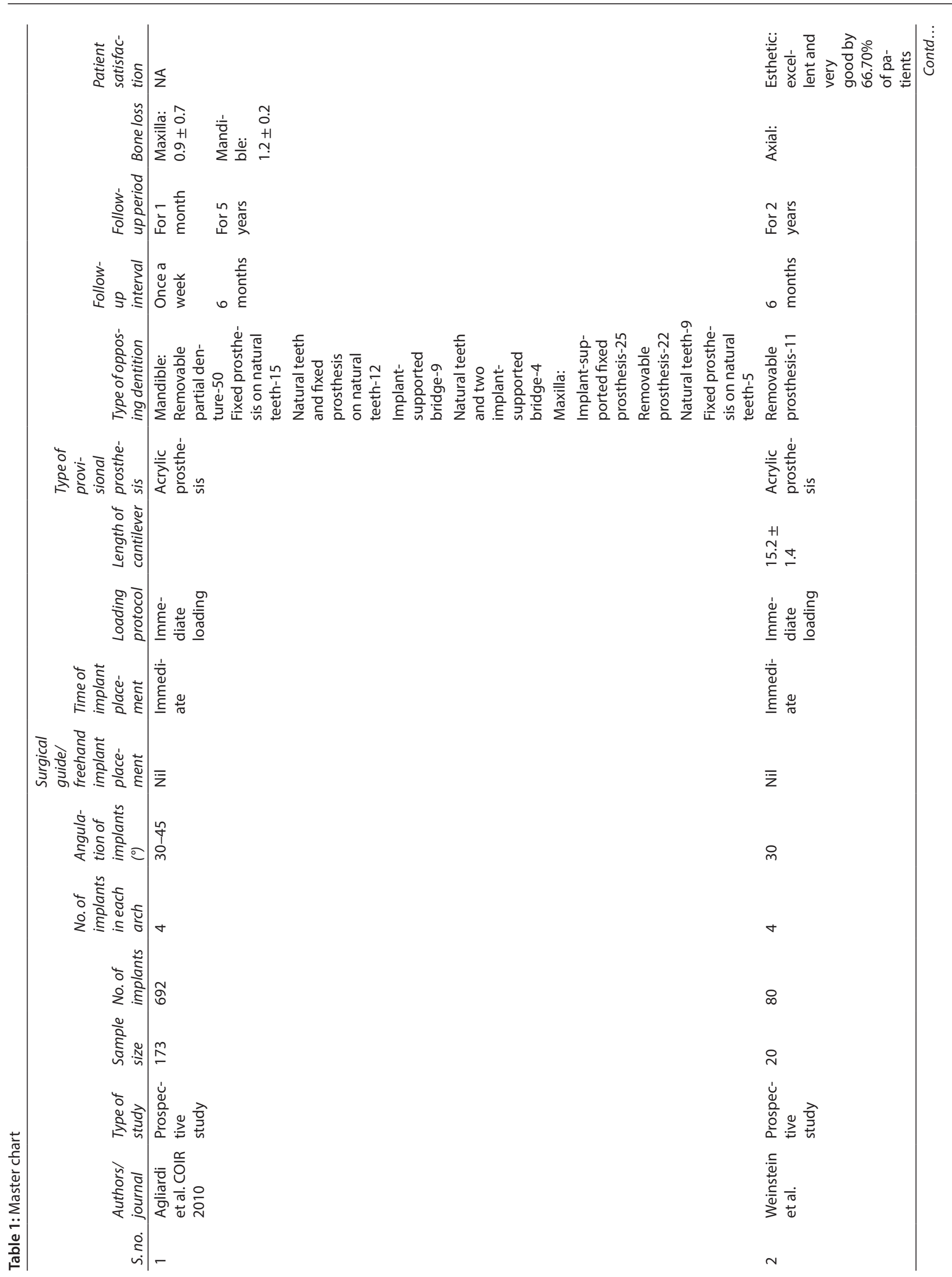




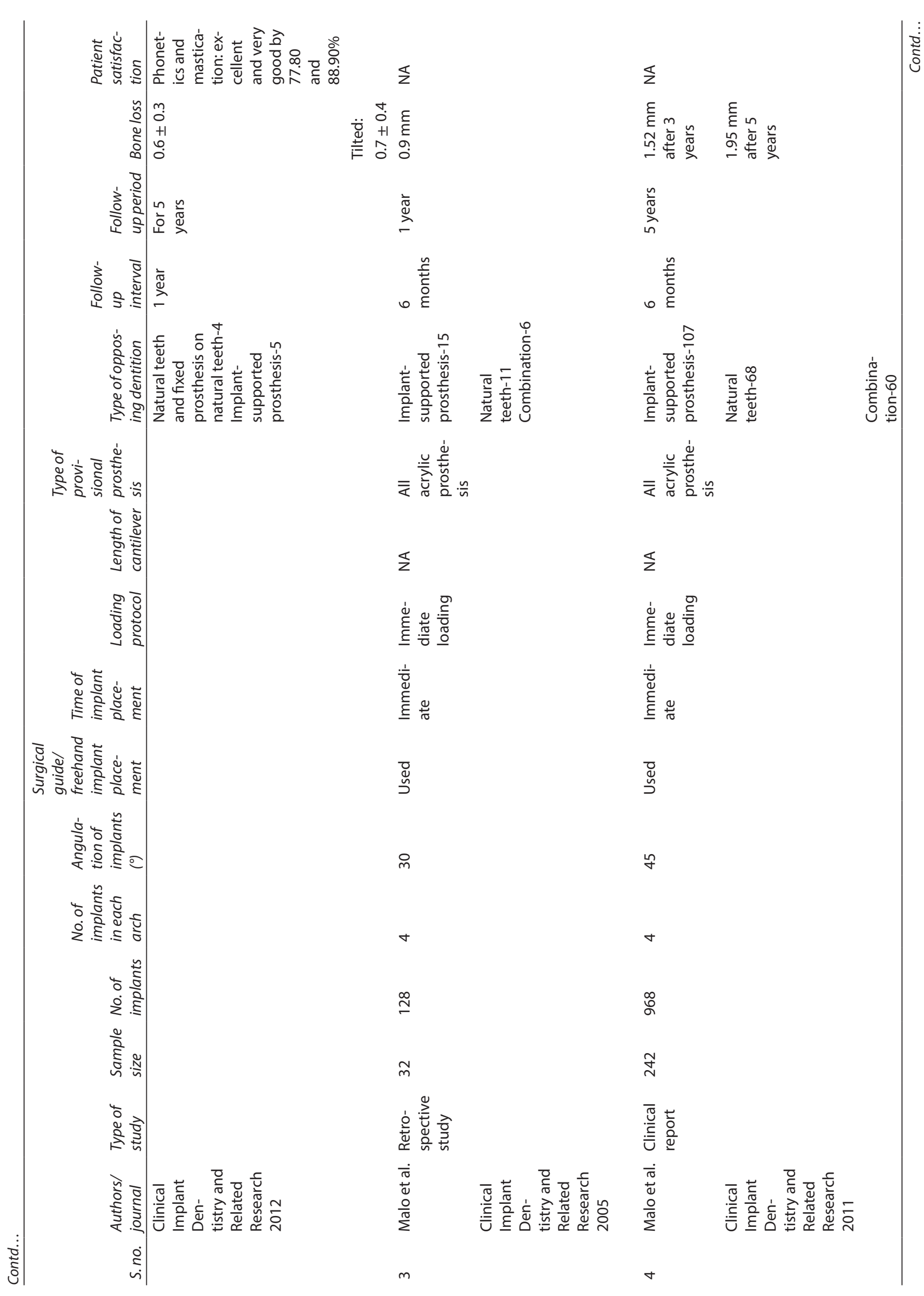




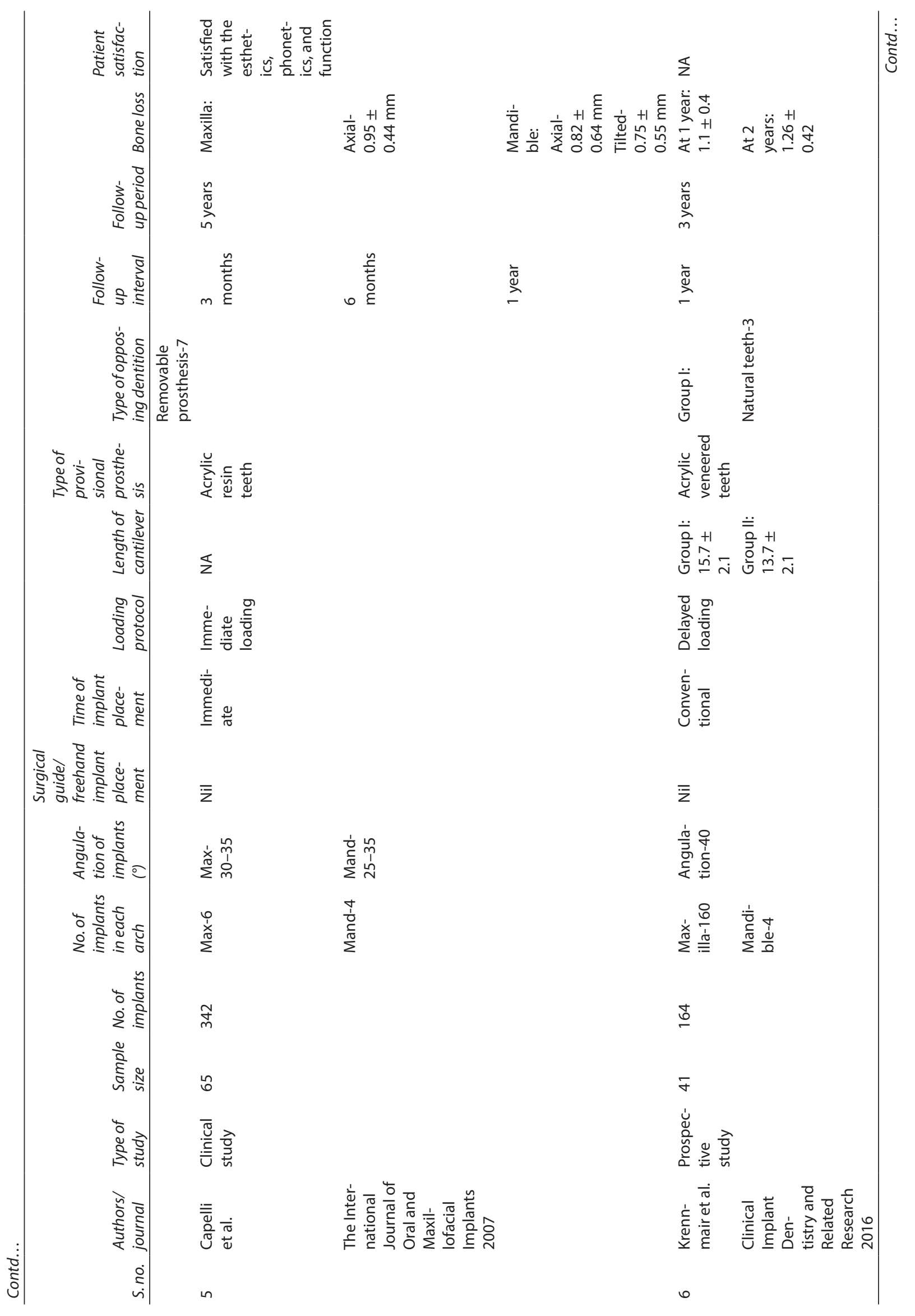




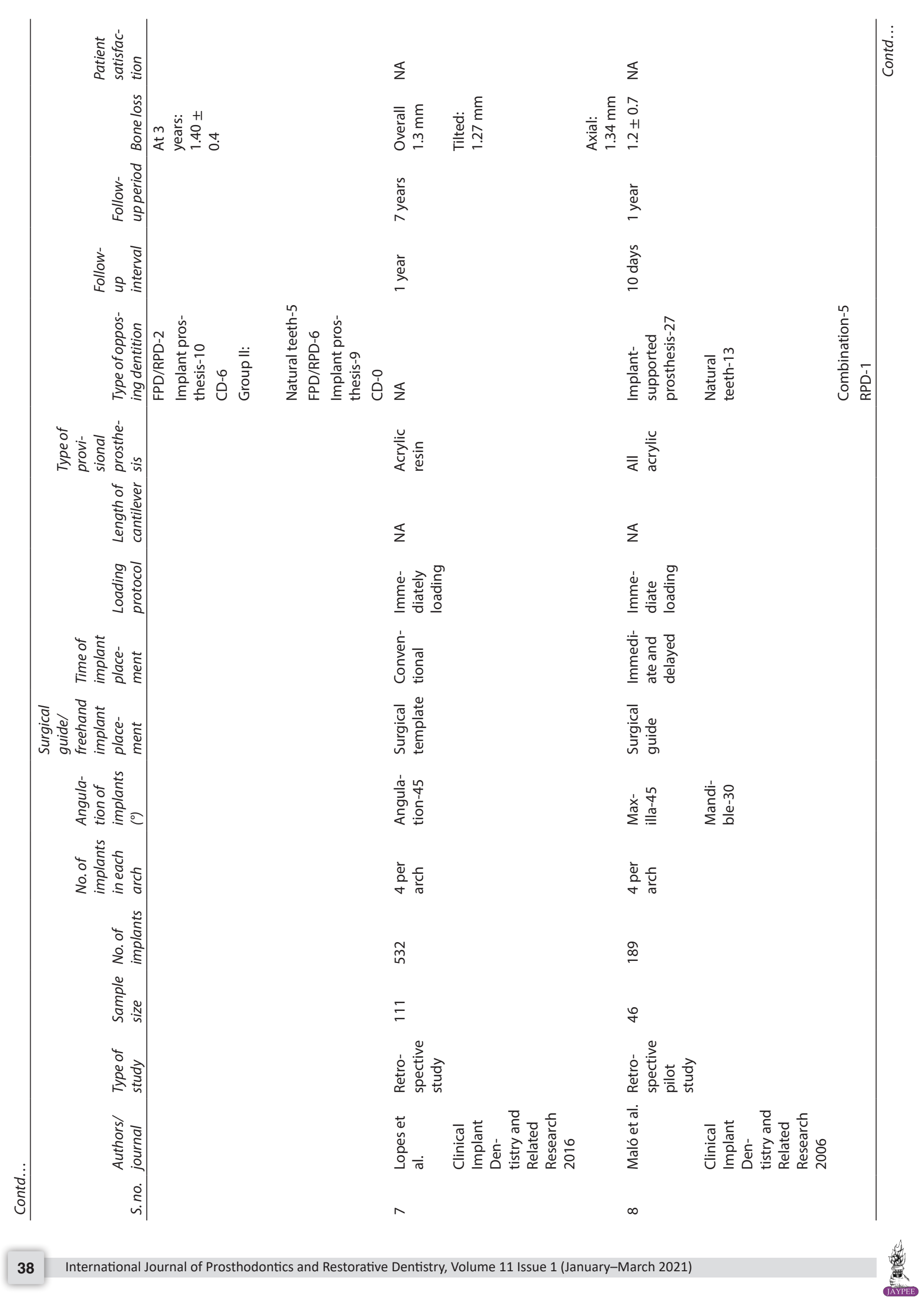




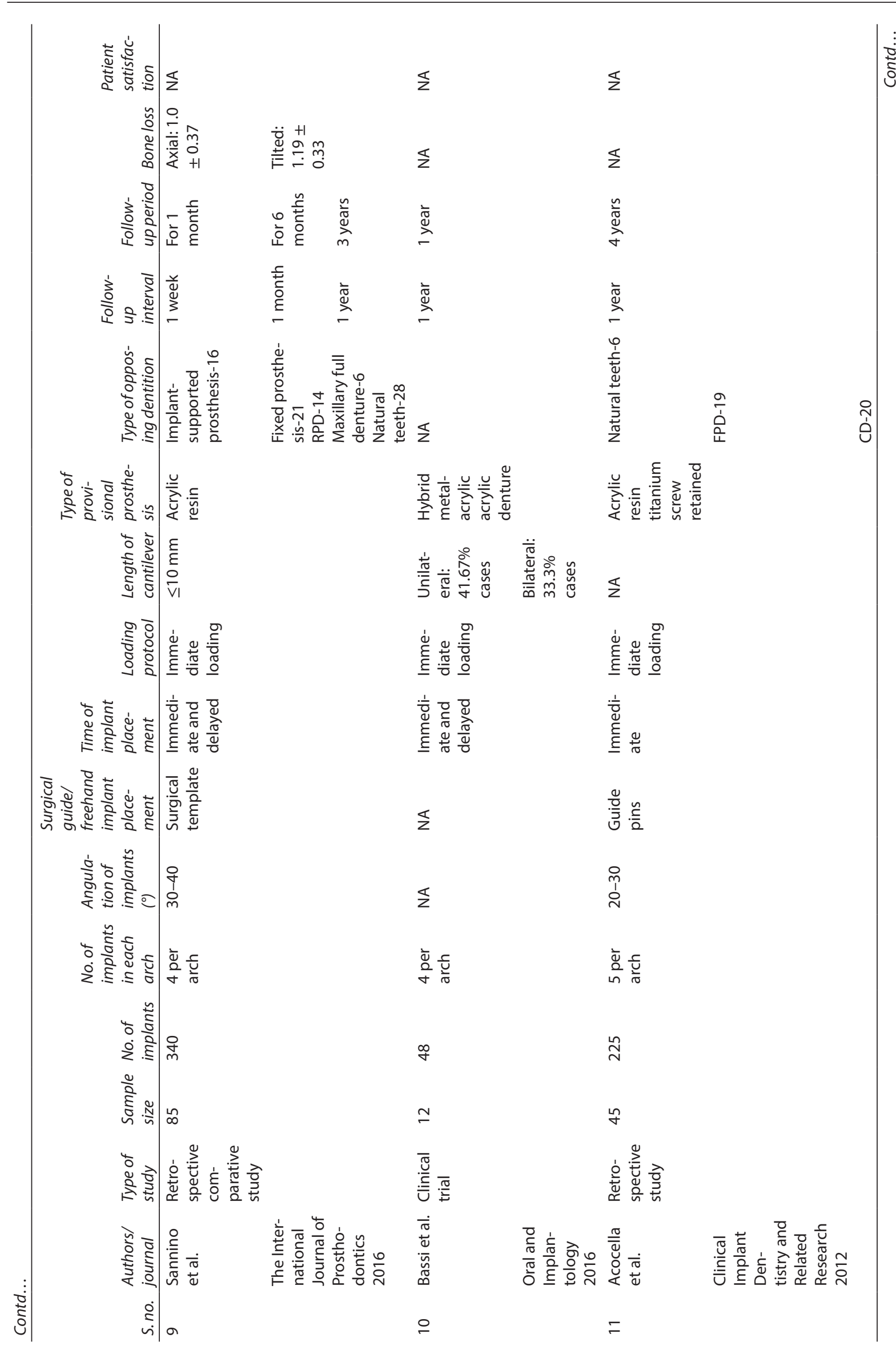




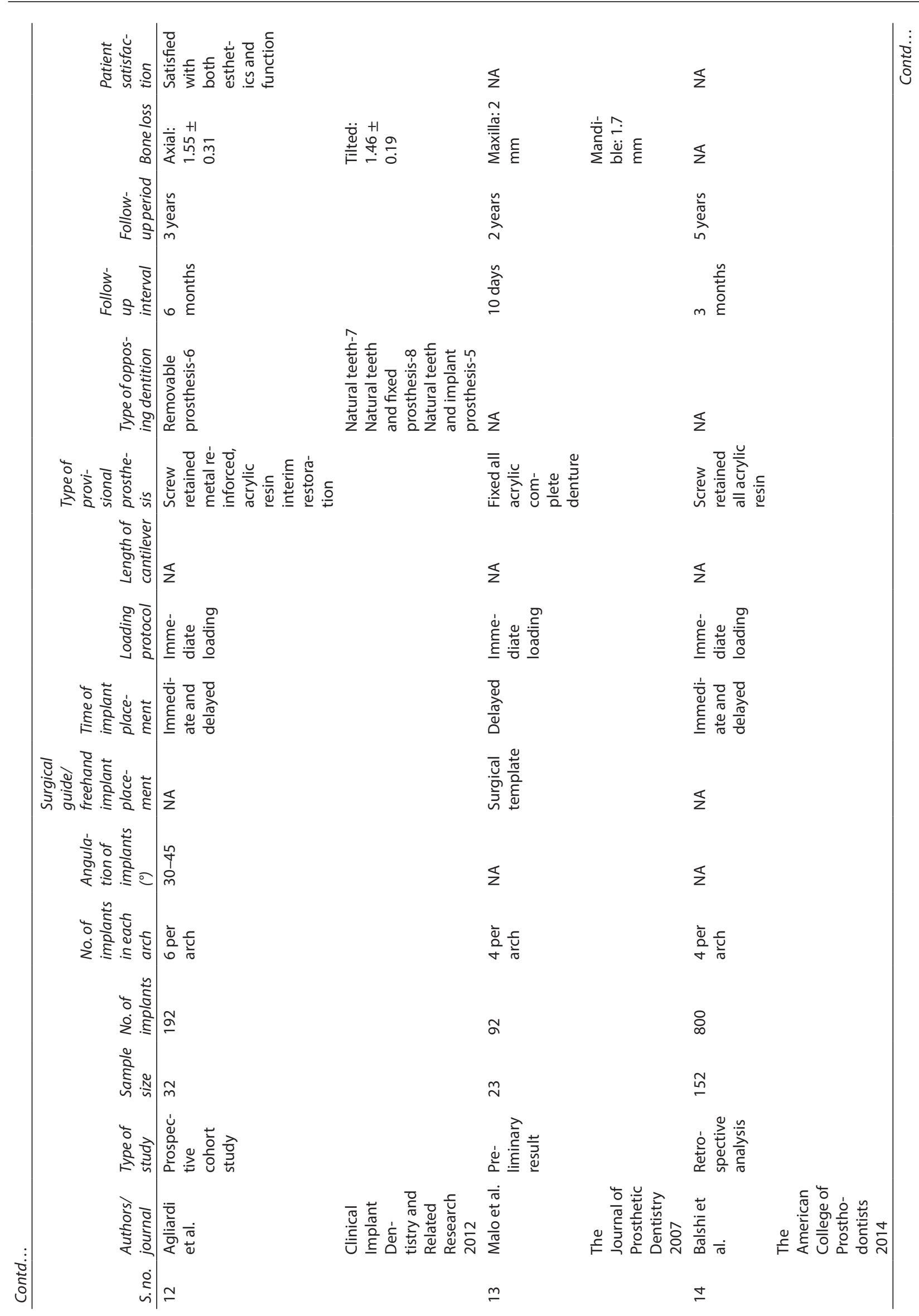




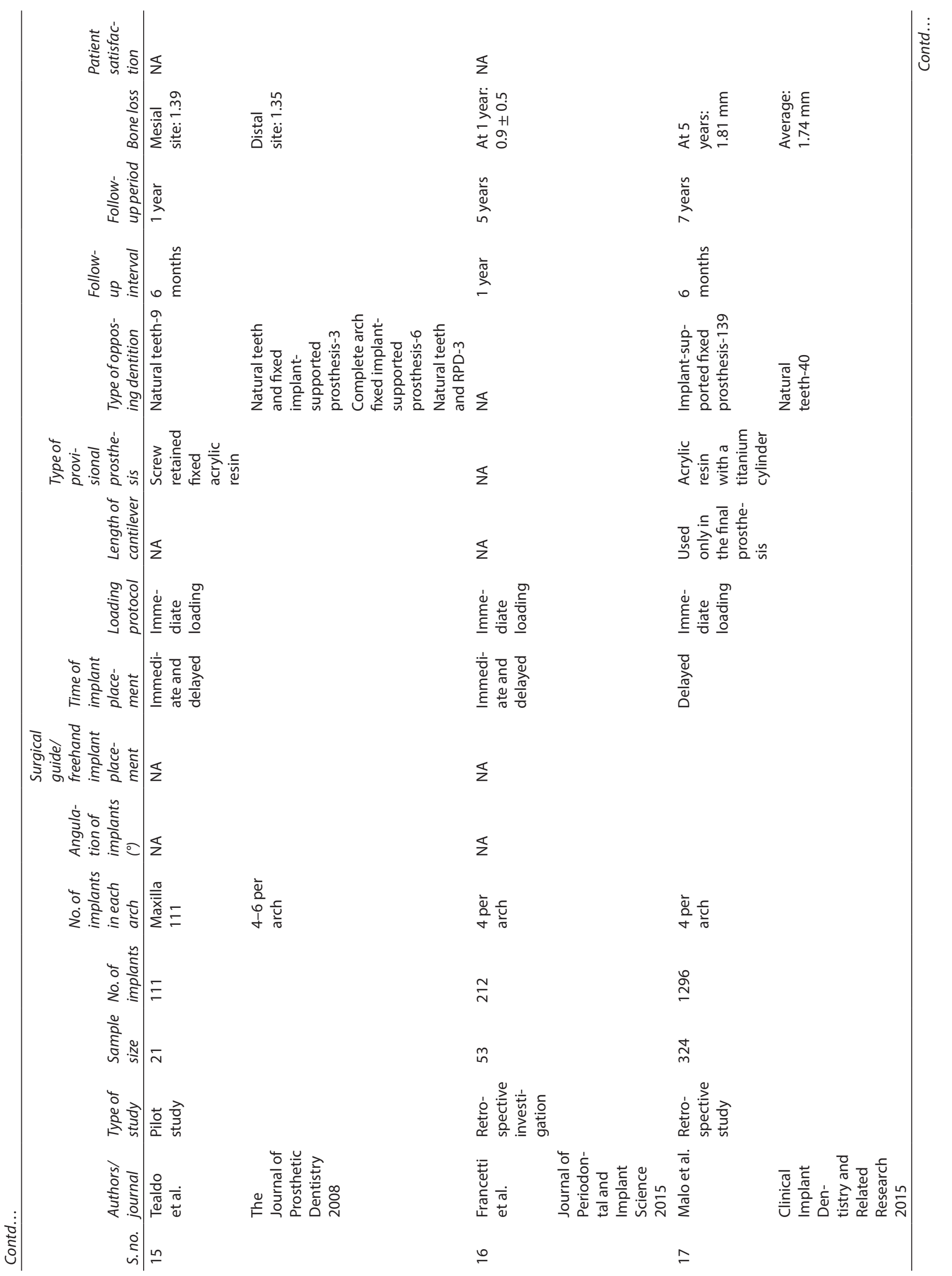




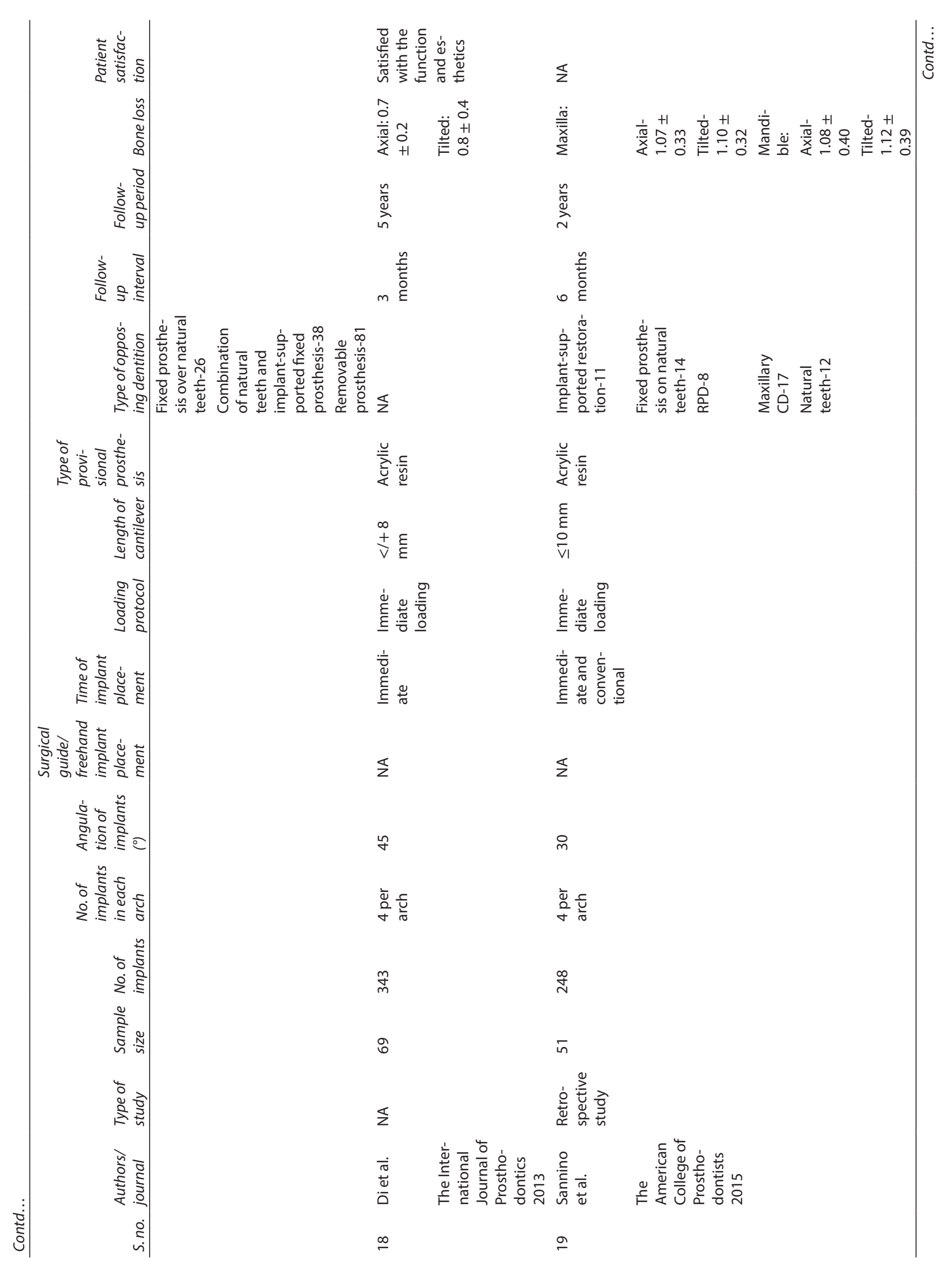




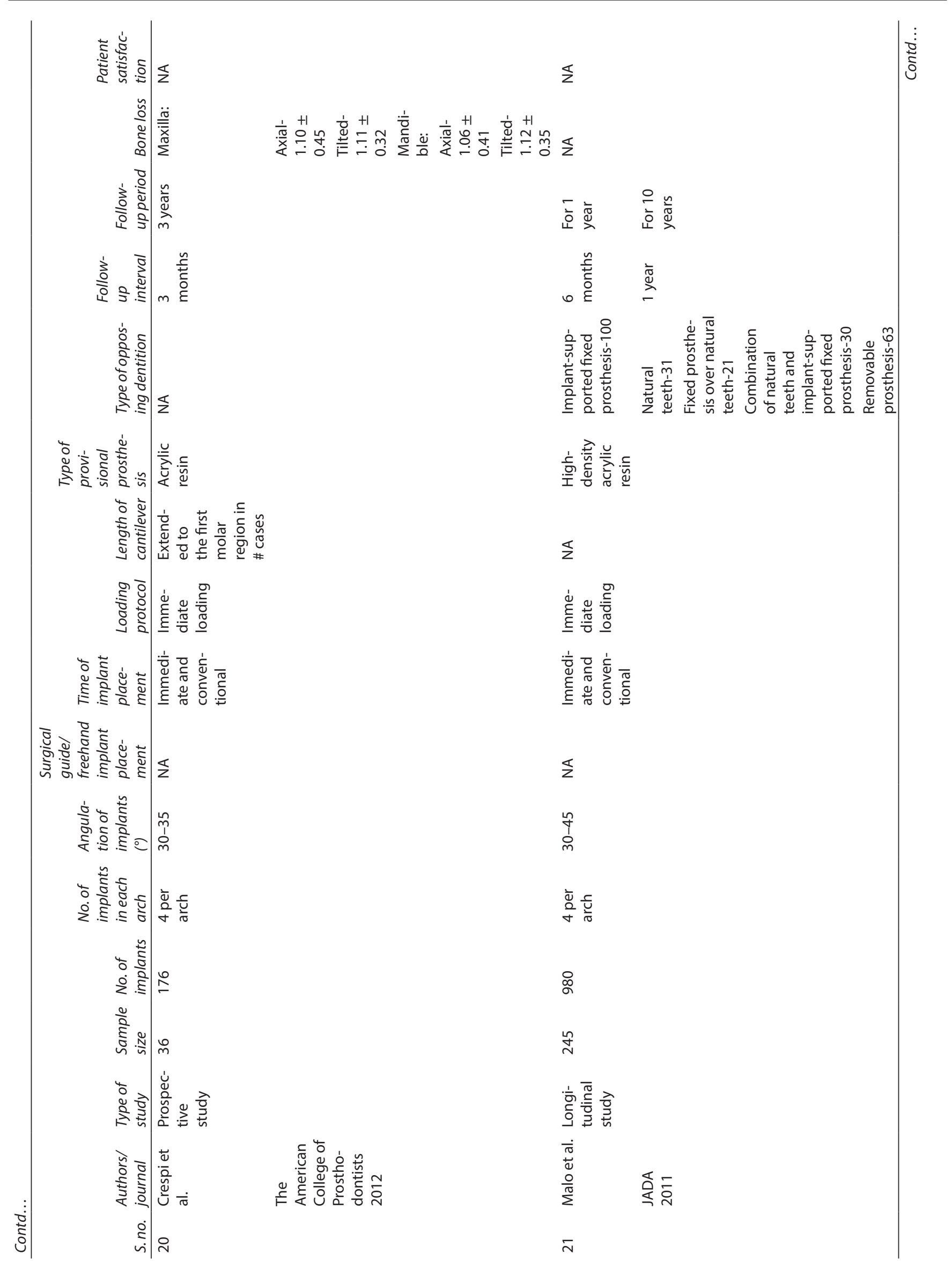




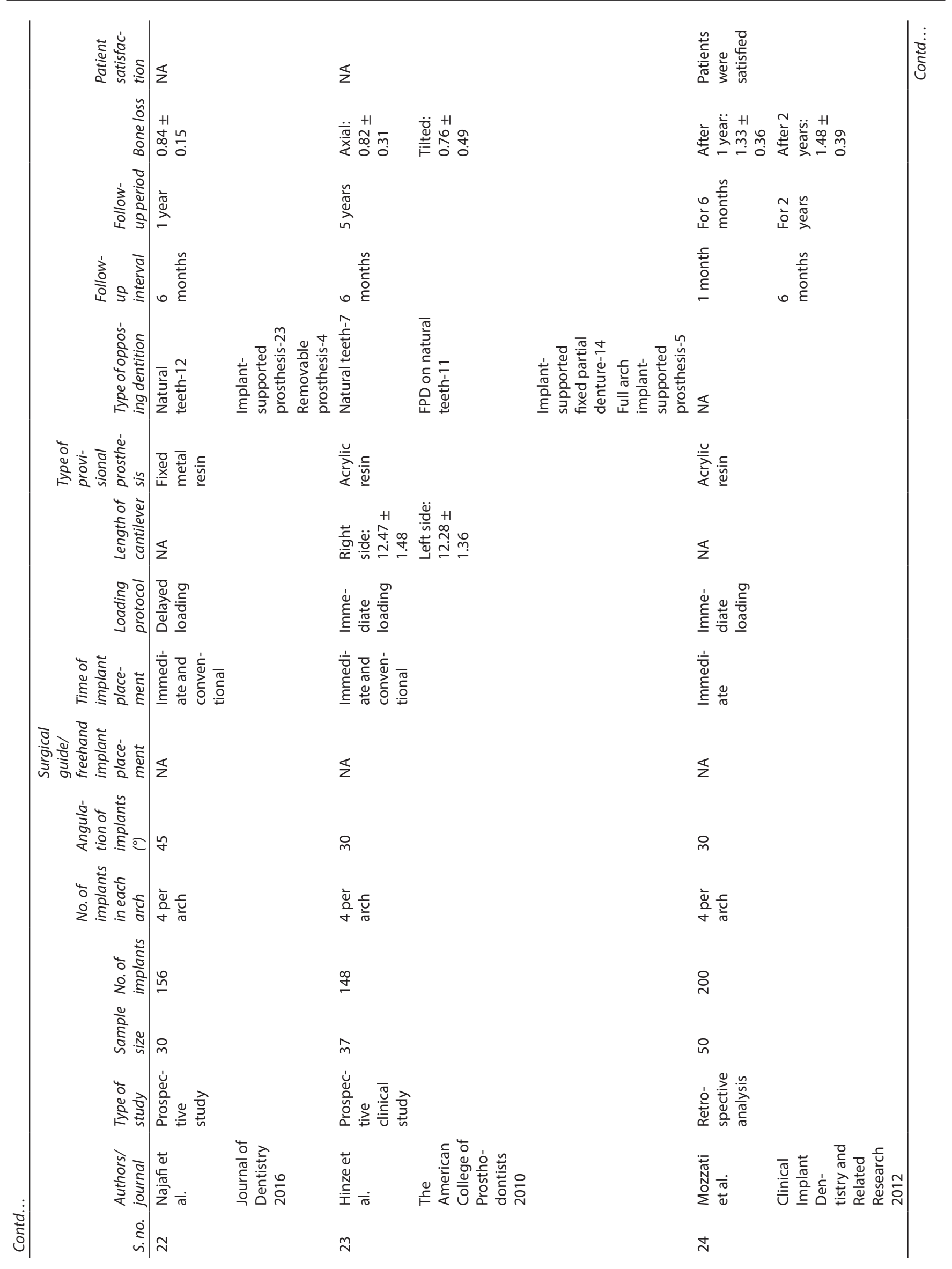




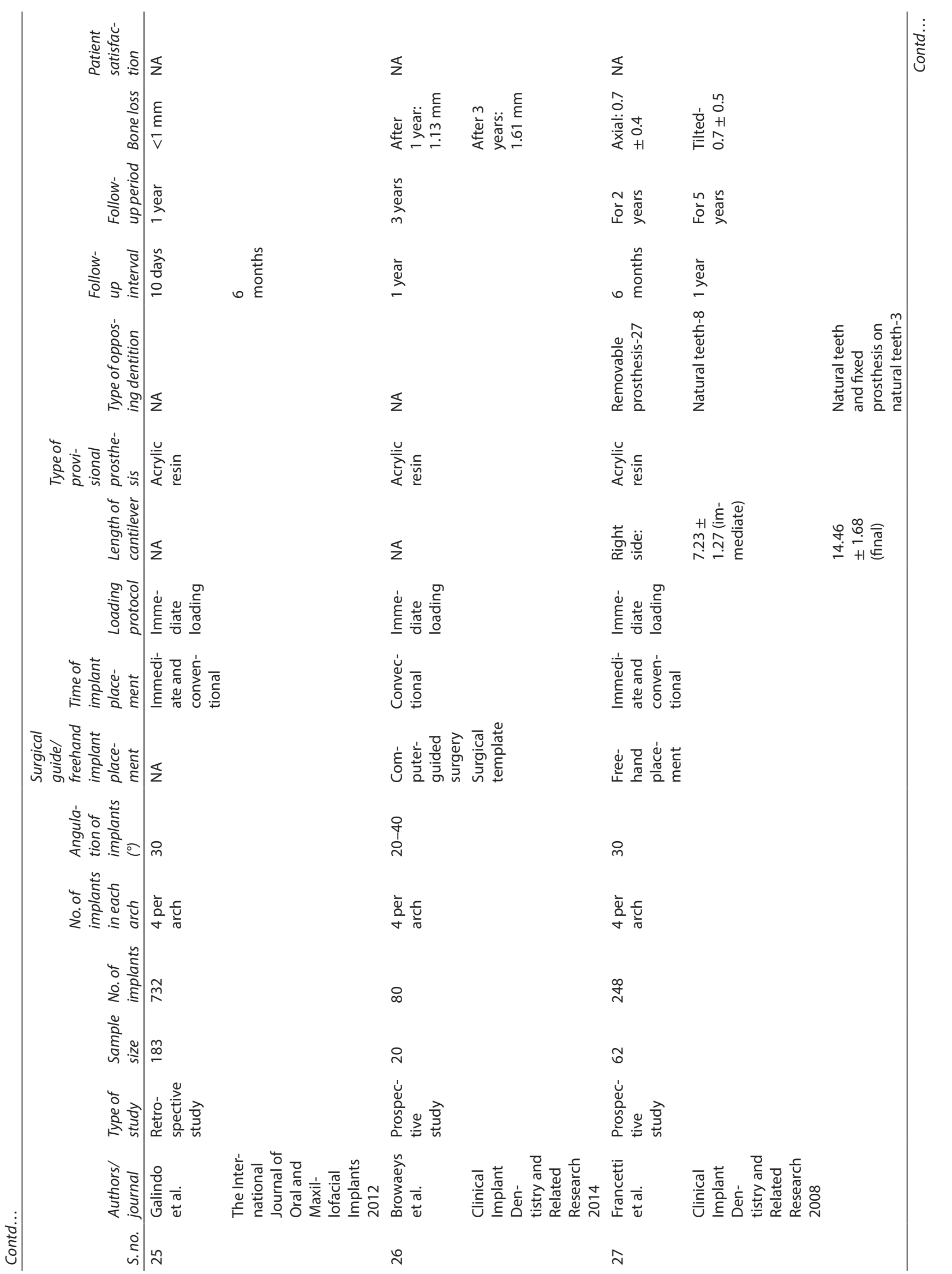




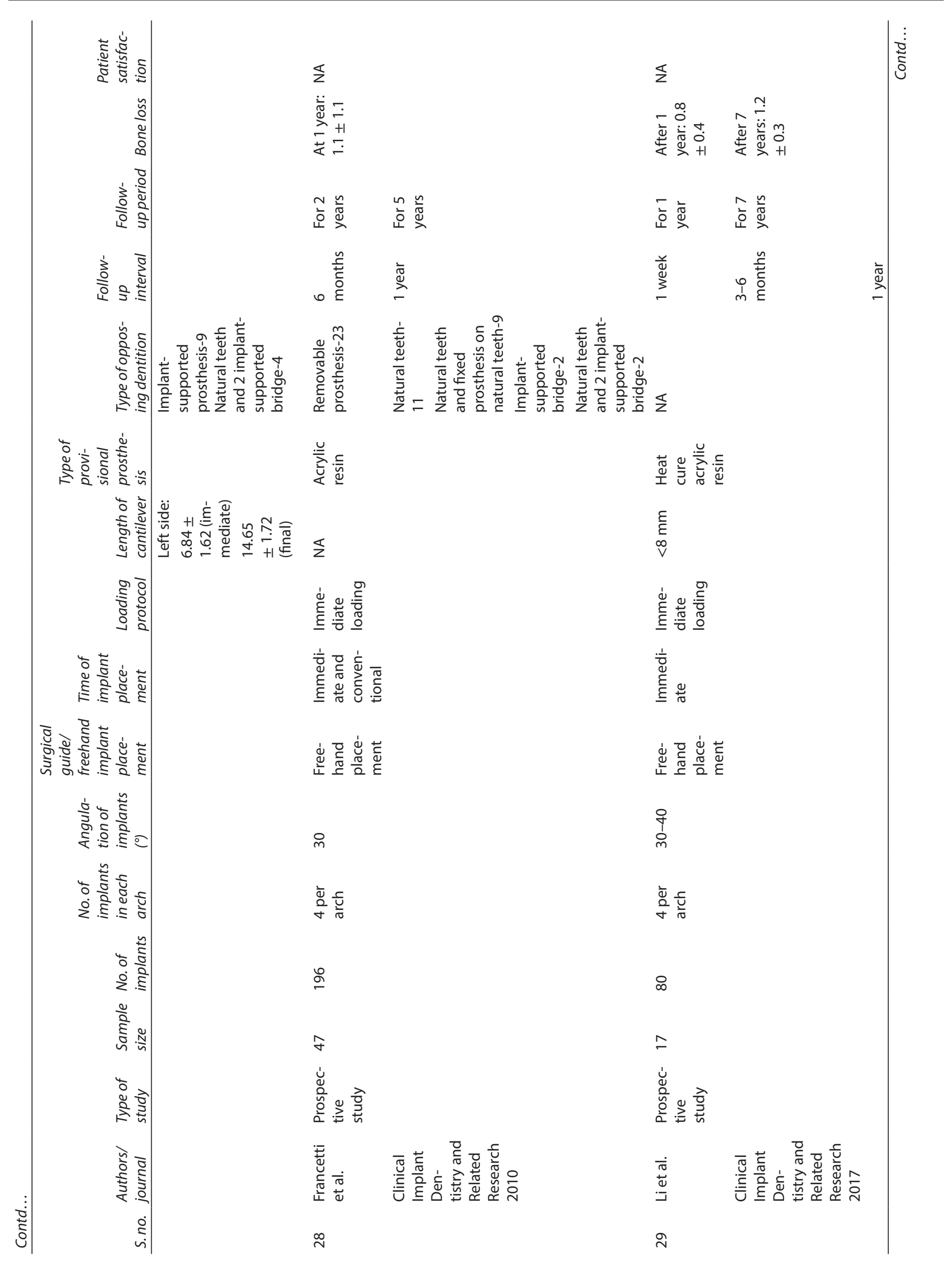




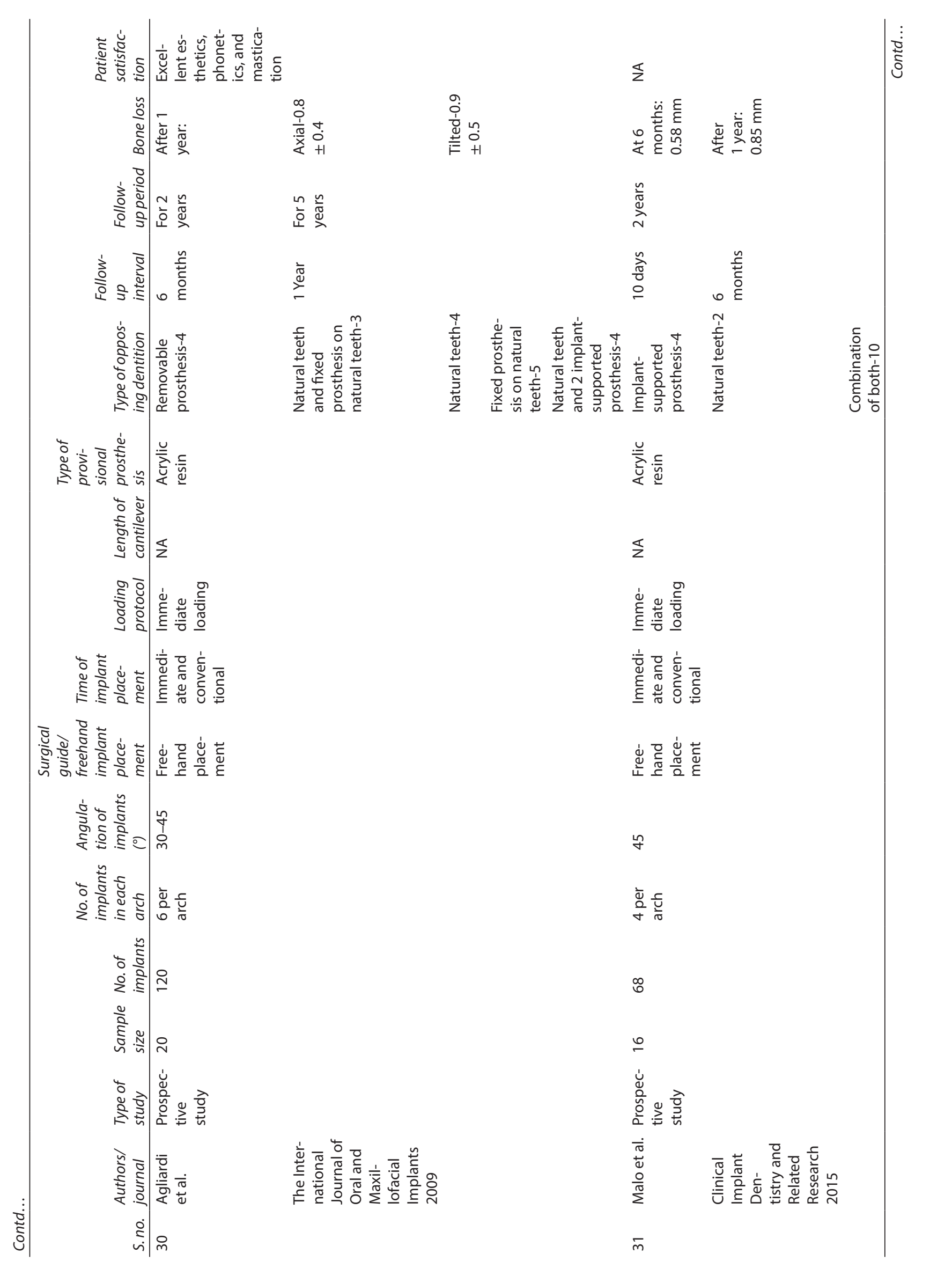




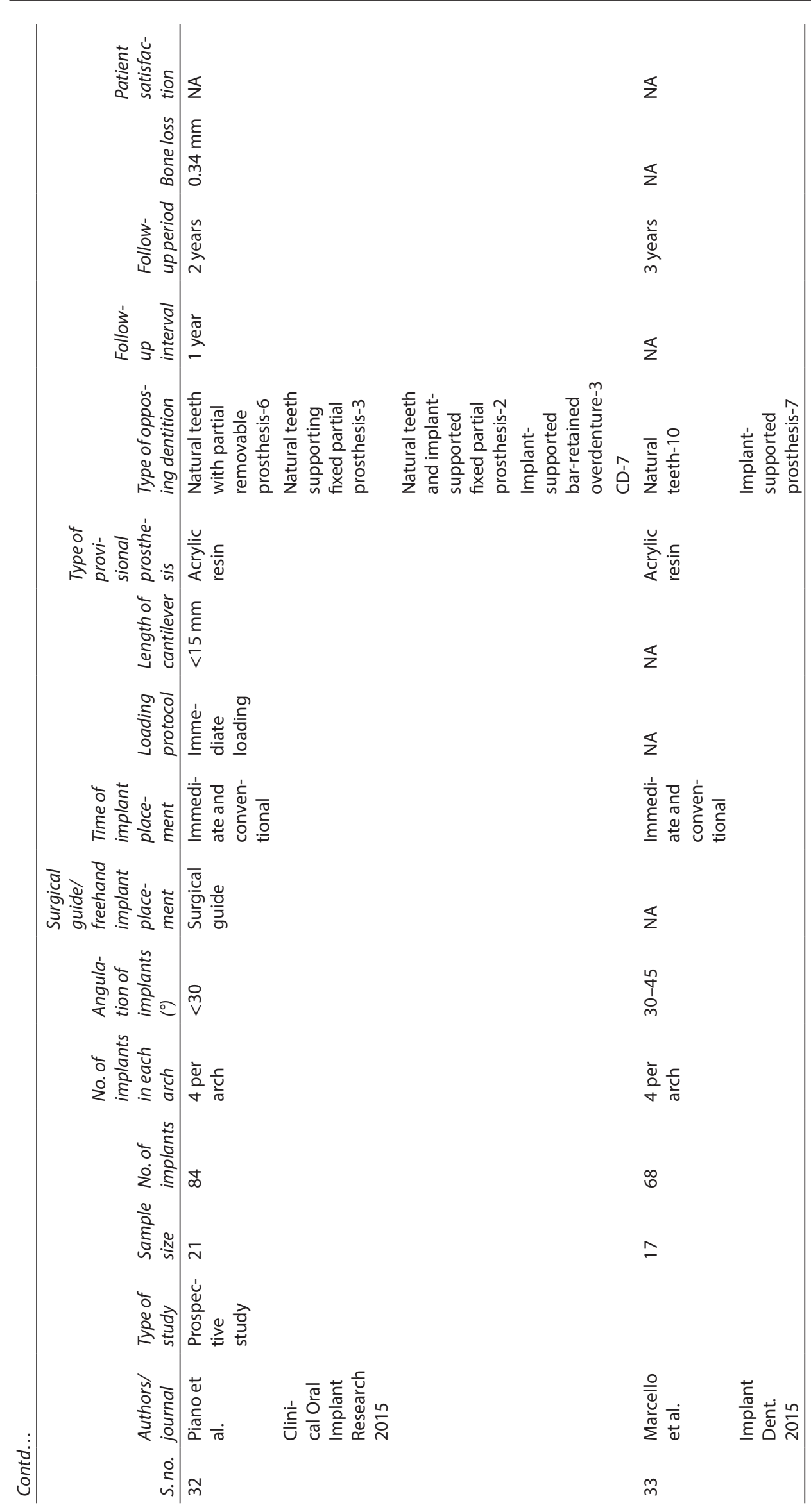




\begin{tabular}{|c|c|c|c|c|}
\hline S. no. & Type of study & & Number & Percentage \\
\hline \multirow[t]{2}{*}{1} & Prospective & Clinical & 14 & 42.42 \\
\hline & & Cohort & & \\
\hline \multirow[t]{3}{*}{2} & Retrospective & Pilot & 11 & 33.33 \\
\hline & & Investigation & & \\
\hline & & Comparative & & \\
\hline 3 & Clinical report & & 1 & 3.03 \\
\hline 4 & Clinical study & & 1 & 3.03 \\
\hline 5 & Clinical trial & & 1 & 3.03 \\
\hline 6 & $\begin{array}{l}\text { Preliminary } \\
\text { report }\end{array}$ & & 1 & 3.03 \\
\hline 7 & Pilot study & & 1 & 3.03 \\
\hline 8 & $\begin{array}{l}\text { Longitudinal } \\
\text { study }\end{array}$ & & 1 & 3.03 \\
\hline 9 & Not mentioned & & 1 & 3.03 \\
\hline
\end{tabular}

Table 3: Angulation of implants used

\begin{tabular}{llll}
\hline S. no. & Implant angulation $\left({ }^{\circ}\right)$ & No of articles & Percentage \\
\hline 1 & 30 & 12 & 44.44 \\
2 & 45 & 6 & 22.22 \\
3 & $20-30$ & 1 & 3.70 \\
4 & $20-40$ & 1 & 3.70 \\
5 & $25-35$ & 1 & 3.70 \\
6 & $30-35$ & 2 & 7.40 \\
7 & $30-40$ & 2 & 7.40 \\
8 & $30-45$ & 5 & 18.51 \\
\hline
\end{tabular}

at an angulation. The orientation of 60 (37.27\%) implants was not mentioned.

In the maxillary arch, 93 implants (57.76\%) failed, out of which 19 (11.80\%) implants were axially placed, and 38 (23.60\%) implants were tilted. The angulation of the remaining 36 (22.36\%) implants was not mentioned.

In the mandibular arch, a total of 60 (37.27\%) implants failed, out of which 5 (3.11\%) implants were axially placed and 10 (6.21\%) implants were tilted. The angulation of 45 (27.95\%) implants was not specified.

The position and angulation of 8 (4.97\%) implants were not specified (Table 3).

\section{Discussion}

\section{Article Type}

Out of the 75 articles obtained 33 were selected which fulfilled all the inclusion criteria.

Fourteen ${ }^{2,23,25-36}$ articles (42.424\%) were prospective studies of which one (7.14\%) was a clinical study ${ }^{30}$ and one $(7.14 \%)$ a cohort study. ${ }^{27}$ Eleven articles ${ }^{1,20,24,37-43}(33.33 \%)$ were retrospective studies including one (9.09\%) pilot study, ${ }^{38}$ one (9.09\%) investigation ${ }^{41}$ and one $(9.09 \%)$ comparative study. ${ }^{39}$ There was also a clinical report $(3.03 \%),{ }^{44:}$ clinical study $(3.03 \%),{ }^{22}$ clinical trial $(3.03 \%),{ }^{3}$ preliminary report (3.03\%), ${ }^{45}$ pilot study (3.03\%), ${ }^{46}$ and a longitudinal study
(3.03\%). ${ }^{47}$ Two (6.060\%) articles did not mention the type of study conducted. ${ }^{48,33}$ The majority of the articles selected for this review were prospective studies as these types of studies help the clinician to accurately catalog the progress of the treatment.

\section{Study Population}

A total of 2,398 patients were studied of which 967 (40.681\%) were males and 1,235 (51.956\%) were females. One hundred and ninetysix (8.17\%) did not identify the gender of the patients. An average of 72.67 participants was present in each study. The highest sample size was found to be 324 in the article by Paulo Maló, Miguel de Araújo Nobre, Armando Lopes, Ana Ferro, Inês Gravito ${ }^{24}$ and the lowest was 12 by Bassi, Andrisani, Lico, Ormanier, Arcuri. ${ }^{3}$ The highest number of male participants in a single study was found to be $130^{24}$ and the lowest was $4 .^{33}$ The highest number of female participants in a single study was $194^{24}$ and the lowest 7.33,35 The oldest participant was aged 89 years old and the youngest was 23 years old. The average age of the patients was 57.98 years, which shows that the full mouth implant rehabilitation is not necessarily indicated just for the geriatric or younger age group but can be applied to all age groups. The large sample size helps to get statistically significant results and authenticate the treatment protocol.

\section{Number of Implants Placed}

A total of 10,300 implants were placed of which 3,489 (33.87\%) were placed in the maxillary arch and 6,045 (58.68\%) in the mandibular arch. The location of the remaining $766(7.44 \%)$ implants placed were not specified. A total of 5,069 implants (49.21\%) were tilted and $5,116(49.66 \%)$ were axial. The maximum number of implants placed in a single study was $1,296(12.58 \%)^{24}$ and the minimum number of implants placed was $48(0.46 \%){ }^{3}$ The maximum number of implants placed in the maxillary arch in a single study was $968(0.093 \%)$ and $1,296(12.58 \%)^{24}$ in the mandibular arch. The minimum number of implants used in a study was $36(0.35 \%)$ in the maxillary arch and $4(0.04 \%)$ in the mandibular arch.

\section{Number of Implants Placed Per Arch}

Twenty-eight (84.84\%) authors ${ }^{1-3,20,23-26,28-33,35-39,41-45,47,33}$ placed four implants per arch, two (6.06\%) articles ${ }^{22,46}$ placed four to six implants, and one (3.03\%) article ${ }^{40}$ placed five implants per arch. These 31 (93.93\%) authors placed two implants at an angulation. Additionally, two (6.06\%) authors ${ }^{27,34}$ placed six implants per arch of which four implants were tilted.

\section{Implant Angulation}

The articles used in this review placed the implants in axial positions as well as in different angles. Few studies placed the implants in multiple angles and few used a specific angle for the implant placement. Twelve (44.44\%) articles ${ }^{20,23-25,30-32,36,38,42,43}$ placed the implants at $30^{\circ}$ and six (22.22\%) articles $29,35,37,38,44,48$ placed the implants at $45^{\circ}$. One (3.70\%) article $e^{40}$ placed the implants at angulations between 20 and $30^{\circ}$, one (3.70\%) article ${ }^{31}$ between 20 and 40 , one (3.70\%) article 22 between 25 and 35, two (7.40\%) articles $^{22,28}$ between 30 and 35 , two (7.40\%) articles ${ }^{33,39}$ between 30 and 40 , and five (18.51\%) articles $2,27,34,47,33$ between 30 and 45 . Six (22.22\%) articles ${ }^{1,3,26,41,45,46}$ did not mention the angulation at which the implants were placed. The placement of implants in 
different angulations is an important factor that helps to identify the most favorable angulation which is to be used for the success of the all-on- 4 and all-on- 6 treatment protocols.

\section{Use of Surgical Guide}

Four (12.12\%) studies ${ }^{31,37,39,45}$ used a surgical template for surgery using a flapless approach and three $(9.09 \%)$ studies $^{35,17,20}$ used a surgical template with the flap elevation technique. Twenty-six (78.78\%) studies did not use surgical templates and employed a freehand implant placement approach.

\section{Time of Implant Placement}

Immediate, delayed, and a combination of the two were practiced. Two (6.06\%) authors ${ }^{33,43}$ used an immediate placement of implants into the arch after extraction and five (18.51\%) authors $24,26,31,37,45$ used a delayed protocol for the placement of the implants. A combination of delayed and immediate implant placement was done by 26 (78.78\%) authors.

\section{Loading Protocol}

The loading protocol followed by all the authors were different. Six (18.18\%) authors $1,3,37,39,42,45$ loaded the implants immediately after surgery. Four (12.12\%) authors $2,20,25,27$ loaded the implants 3 hours after surgery, four (12.12\%) authors $35,38,43,47$ after 2-3 hours, two $(6.06 \%)$ authors ${ }^{27,34}$ after 4 hours, and two $(6.06 \%)$ authors ${ }^{45,48}$ after 6 hours. One article ${ }^{30}$ (3.03\%) loaded the implants within 24 hours after surgery and two (6.06\%) articles loaded 24 hours after the surgery. One article (3.03\%) loaded implants within 48 hours $^{31}$ and seven (21.21\%) after 48 hours. ${ }^{22,23,25,32,36,40,41}$ One article (3.03\%) loaded the implants between 8 hours and 48 hours after the surgery, one article (3.03\%) after 3 days of surgery, ${ }^{29}$ and one (3.03\%) 2 months after the surgery. ${ }^{26} \mathrm{~A}$ single article (3.03\%) loaded the implants on the same day of the surgery. ${ }^{24}$ One article ${ }^{33}$ (3.03\%) did not mention the loading protocol followed.

\section{Presence and Length of Cantilever}

The use of cantilever was assessed and it was found that 21 (63.63\%) articles ${ }^{1,2,20,22-26,28,30,32,33,36,39,40,42-44,46-48}$ declared the use of cantilever during restoration and 12 (36.36\%) did not use cantilever during restoration. ${ }^{3,27,29,31,34,35,37,38,41,43,45,33}$ The longest cantilever used was of $15.20 \mathrm{~mm}$ in length ${ }^{25}$ and the shortest was of $6.84 \mathrm{~mm}$ in length. ${ }^{23}$ The use of cantilever should be avoided in complete arch replacement or should be kept to not $>2$ times the anteroposterior spread or a maximum of $20 \mathrm{~mm}$.

\section{Type of Provisional Restoration/Prosthesis}

The type of provisional or temporary restoration was taken into consideration. Eleven (33.33\%) articles gave an acrylic resin prosthesis. Five (15.15\%) articles gave an acrylic prosthesis, three (9.09\%) articles gave a screw-retained acrylic prosthesis and two (6.06\%) articles gave acrylic prosthesis without a metal framework. Three $(9.09 \%)$ studies placed all-acrylic prostheses. One $(3.03 \%)$ study gave acrylic resin teeth to the patients and another gave acrylic veneered teeth. Acrylic dentures were given in one study and fixed acrylic resin complete denture was given in another study. One study had given acrylic resin with titanium cylinder. Acrylic resin prosthesis with the screw-retained bar-retained restoration was placed in one study. Fixed metal resin prosthesis was placed in an article and heat-cured acrylic resin prosthesis in another study. The type of provisional restoration placed influences the load on the implants which has an impact on the success of the implant treatment.

\section{Type of Opposing Dentition}

When the type of opposing dentition was considered it was found that in 311 cases the prosthesis was opposed by the natural dentition. Thirty-nine cases had a combination of natural teeth and fixed prosthesis on the natural dentition. Three hundred and twenty-seven cases had a removable prosthesis opposing the implant-supported prosthesis and 513 cases had implant-supported prosthesis itself on the opposite arch. Eighty-five cases had fixed prosthesis on natural teeth, three had natural teeth supporting fixed partial prosthesis, and five had natural teeth and implantsupported fixed partial prosthesis. Fifty-six cases reported to have a complete denture in the opposing arch and three cases had an implant-supported bar-retained overdenture. In 154 cases, the opposing arch was a combination of natural teeth and implantsupported prosthesis. Nineteen cases had had implant-supported/ retained prosthesis. Fixed partial dentures were present in 40 cases and implant-supported bridge in 11 cases. Four cases presented with a combination of natural teeth and 2 implant-supported fixed partial dentures and 10 cases a combination of natural teeth with 2 implant-supported bridges. A combination of removable partial dentures supported by natural teeth was found in 13 cases. Eight cases had fixed partial dentures or removable partial dentures which were not specified by the authors. The type of opposing dentition influences the treatment plan taken for the patient. The forces acting on the implant-supported prosthesis depend on the condition of the opposing dentition. The presence of natural teeth or fixed prosthesis on the opposing arch requires the use of a short cantilever and an increased number of implants. A removable prosthesis on the opposing arch creates a minimum bite force.

\section{Follow-up Interval}

Most of the studies followed the cases at equal time intervals. Few studies followed-up the subjects at short intervals immediately after the treatment and at a longer interval period after some time. Nine (27.27\%) studies followed-up with their patients at an interval of less than a month. Twelve (36.36\%) articles followed-up with their patients at an interval of $1-3$ months. Twenty $(60.60 \%)$ articles also had a follow-up interval of 4-6 months and 22 (66.67\%) of the studies had followed up the patients at an interval between 7 months and 12 months. The follow-up interval used by few articles is not singular. Few studies had shorter follow-up intervals in the initial few months to a year of the beginning of the treatment which was subsequently increased to extended intervals in the subsequent years. A follow-up interval of 1 month for the initial year of treatment is reasonable. The interval in the subsequent years can be of a longer duration of 6 months to 1 year.

\section{Follow-up Period}

The articles selected for this study had a follow-up period of not less than a year. Eleven (33.33\%) studies $3,20,29,35,36,38,42,43,45,46$ followed up their patients for a period of 1 to 2 years after implant placement. Seven (21.21\%) articles ${ }^{26-28,31,39,40,33}$ had followed up their cases for

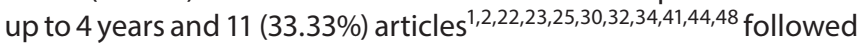
up their cases for 5-6 years following implant placement. About 
Flowchart 1: Article search methodology

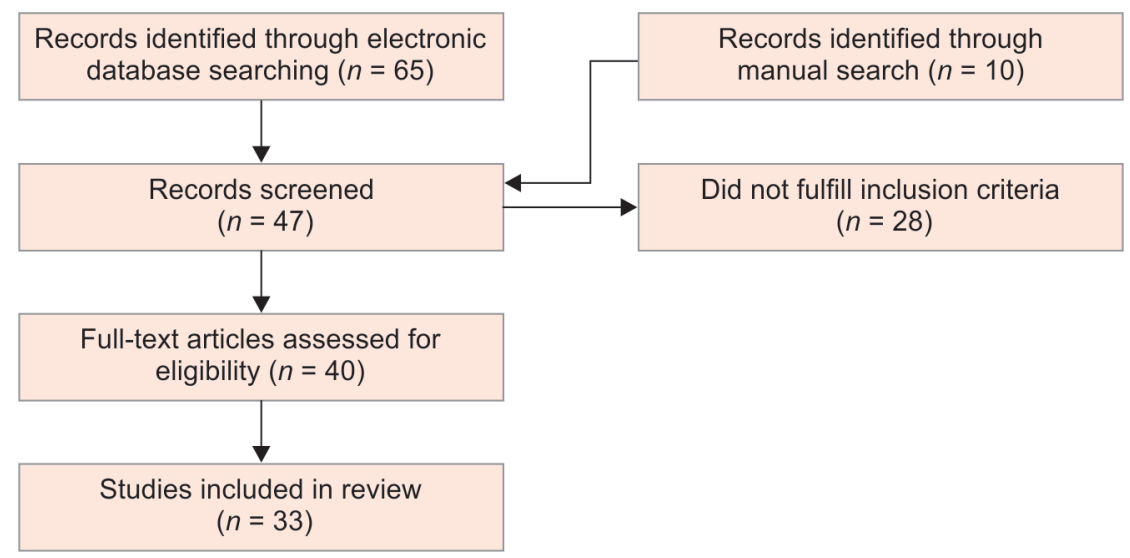

four (12.12\%) studies ${ }^{24,33,37,47}$ maintained patient follow-up for 7 or more years. Patient follow-up helps to carefully evaluate the time of implant failure. The failure of the implant is not a time dependent factor, but most of the implants which failed in the studies did so in the first 6 months after placement (Flowchart 1).

\section{Bone Loss}

An implant is said to be a failure if the peri-implant bone loss is $>1$ $\mathrm{mm}$ in the first year after the placement of an implant. The bone loss in the subsequent years should not be $>0.2 \mathrm{~mm}$ per year. Twenty-five (0.24\%) implants reported a peri-implant bone loss of $>1 \mathrm{~mm}$. In the maxillary arch, five (20.00\%) implants that lost $>1 \mathrm{~mm}$ peri-implant bone were axially placed and five (20.00\%) were placed at an angulation. In the mandibular arch, six (24.00\%) implants showed bone loss $>1 \mathrm{~mm}$ in which two (8.00\%) were axially placed implants and two (8.00\%) were tilted implants showed bone loss $>1 \mathrm{~mm}$. The angulation and location of nine (36.00\%) implants were not mentioned in the articles.

\section{Patient Satisfaction}

All-on-4 and all-on- 6 treatment protocol is one of the widely accepted treatment protocols for the rehabilitation of edentulous patients with high levels of patient satisfaction. Six (18.18\%) of the

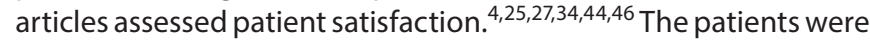
completely satisfied with the esthetic and functional outcome of the prosthesis. The ultimate aim of all prosthodontic treatments is patient satisfaction in terms of their appearance, functionality, and comfort of the prosthesis.

\section{ConcLusion}

The following conclusions were derived from the findings of this review:

- The survival rate of tilted implants at the implant and prosthetic levels is good.

- There is no significant difference between the survival rate of tilted and axial implants at implant and prosthetic levels.

- Tilted implants provide greater surface area for osseointegration, provide greater primary stability, reduce cantilever length, reduce bone resorption, and also reduce the need for bone grafting.
- The angulation of the implant which provided the most success was found to be $30^{\circ}$.

\section{References}

1. Balshi TJ, Wolfinger GJ, Slauch RW, et al. A retrospective analysis of 800 Brånemark System implants following the All-on-Four ${ }^{\mathrm{TM}}$ protocol. J Prosthodont 2014;23(2):83-88. DOI: 10.1111/jopr.12089.

2. Agliardi E, Panigatti S, Clerico M, et al. Immediate rehabilitation of the edentulous jaws with full fixed prostheses supported by four implants: interim results of a single cohort prospective study. Clin Oral Implants Res 2010;21(5):459-465. DOI: 10.1111/j.16000501.2009.01852.x.

3. Bassi MA, Andrisani C, Lico S, et al. Upper full arch rehabilitation with sinus by-pass with tilted implants via tapered-threaded expanders in low density bone: a clinical trial. Oral Implantol 2016;9(2):61.

4. Marchesi M, Ferrari C, Superbi S, et al. Modified protocol of the intraoral welding technique for immediate implant-supported rehabilitation of the edentulous maxilla. Implant Dentis 2015;24(1):110-116. DOI: 10.1097/ID.0000000000000189.

5. Shackleton JL, Carr L, Slabbert JC, et al. Survival of fixed implantsupported prostheses related to cantilever lengths. J Prosthe Dentis 1994;71(1):23-26. DOI: 10.1016/0022-3913(94)90250-X.

6. Goené R, Bianchesi $C$, Hüerzeler $M$, et al. Performance of short implants in partial restorations: 3-year follow-up of Osseotite ${ }^{\oplus}$ Implants. Implant Dentis 2005;14(3):274-280. DOI: 10.1097/01. id.0000173335.90854.d8.

7. Renouard F, Nisand D. Short implants in the severely resorbed maxilla: a 2-year retrospective clinical study. Clin Implant Dent Relat Res 2005;7(s1):s104-s110. DOI: 10.1111/j.1708-8208.2005.tb00082.x.

8. Maló $P$, de Araújo Nobre $M$, Rangert $B$. Short implants placed onestage in maxillae and mandibles: a retrospective clinical study with 1 to 9 years of follow-up. Clin Implant Dent Relat Res 2007;9(1):15-21. DOI: 10.1111/j.1708-8208.2006.00027.x.

9. Khayat $\mathrm{P}$, Nader $\mathrm{N}$. The use of osseointegrated implants in the maxillary tuberosity. Pract Periodont Aesthe Dentis: PPAD 1994;6(4):53-61.

10. Venturelli A. A modified surgical protocol for placing implants in the maxillary tuberosity. Int J Oral Maxillofac Implants 1996;11:743-749.

11. Balshi TJ, Wolfinger GJ, Balshi SF. Analysis of 356 pterygomaxillary implants in edentulous arches for fixed prosthesis anchorage. Int $J$ Oral Maxillofac Implants 1999;14(3):398-406.

12. Brånemark PI, Gröndahl K, Öhrnell LO, et al. Zygoma fixture in the management of advanced atrophy of the maxilla: technique and long-term results. Scandinavian J Plast Reconstruct Surg Hand Surg 2004;38(2):70-85. DOI: 10.1080/02844310310023918. 
13. Galán Gil S, Peñarrocha Diago M, Balaguer Martínez J, et al. Rehabilitation of severely resorbed maxillae with zygomatic implants: an update. Medicina Oral, Patología Oral y Cirugía Bucal (Internet) 2007;12(3):216-220.

14. Aparicio C, Ouazzani W, Hatano N. The use of zygomatic implants for prosthetic rehabilitation of the severely resorbed maxilla. Periodontol 2000 2008;47(1):162-171. DOI: 10.1111/j.1600-0757.2008.00259.x.

15. Maló $P$, de Araujo Nobre M, Lopes I. A new approach to rehabilitate the severely atrophic maxilla using extramaxillary anchored implants in immediate function: a pilot study. J Prosthe Dentis 2008;100(5):354366. DOI: 10.1016/S0022-3913(08)60237-1.

16. Krekmanov L. Placement of posterior mandibular and maxillary implants in patients with severe bone deficiency: a clinical report of procedure. Int J Oral Maxillofac Implants 2000;15(5):722-730.

17. Krekmanov L, Kahn M, Rangert B, et al. Tilting of posterior mandibular and maxillary implants for improved prosthesis support. Int J Oral Maxillofac Implants 2000;15(3):405-414.

18. Aparicio $C$, Perales $P$, Rangert $B$. Tilted implants as an alternative to maxillary sinus grafting: a clinical, radiologic, and periotest study. Clin Implant Dent Relat Res 2001;3(1):39-49.DOI: 10.1111/j.1708-8208.2001. tb00127.x.

19. Maló P, Rangert B, Nobre M. "All-on-Four" immediate-function concept with Brånemark System ${ }^{\circledast}$ implants for completely edentulous mandibles: a retrospective clinical study. Clin Implant Dent Relat Res 2003;5:2-9. DOI: 10.1111/j.1708-8208.2003.tb00010.x.

20. Maló P, Rangert B, Nobre M. All-on-4 immediate-function concept with Brånemark System ${ }^{\circledast}$ implants for completely edentulous maxillae: a 1-year retrospective clinical study. Clin Implant Dent Relat Res 2005;7(s1):s88-s94. DOI: 10.1111/j.1708-8208.2005.tb00080.x.

21. Calandriello R, Tomatis M. Simplified treatment of the atrophic posterior maxilla via immediate/early function and tilted implants: a prospective 1-year clinical study. Clin Implant Dent Relat Res 2005;7(s1):s1-s2. DOI: 10.1111/j.1708-8208.2005.tb00069.x.

22. Capelli M, Zuffetti F, Del Fabbro M, et al. Immediate rehabilitation of the completely edentulous jaw with fixed prostheses supported by either upright or tilted implants: a multicenter clinical study. Int J Oral Maxillofac Implants 2007;22(4):639-644.

23. Francetti L, Agliardi E, Testori T, et al. Immediate rehabilitation of the mandible with fixed full prosthesis supported by axial and tilted implants: interim results of a single cohort prospective study. Clin Implant Dent Relat Res 2008;10(4):255-263. DOI: 10.1111/j.17088208.2008.00090.x.

24. Maló P, de Araújo Nobre M, Lopes A, et al. All-on- $4^{\oplus}$ treatment concept for the rehabilitation of the completely edentulous mandible: A 7-year clinical and 5-year radiographic retrospective case series with risk assessment for implant failure and marginal bone level. Clin Implant Dent Relat Res 2015;17:e531-e541. DOI: 10.1111/cid. 12282.

25. Weinstein R, Agliardi E, Fabbro MD, et al. Immediate rehabilitation of the extremely atrophic mandible with fixed full-prosthesis supported by four implants. Clin Impl Dentis Rela Res 2012;14(3):434-441. DOI: 10.1111/j.1708-8208.2009.00265.x.

26. Krennmair $S$, Weinländer $M$, Malek $M$, et al. Mandibular full-arch fixed prostheses supported on 4 implants with either axial or tilted distal implants: A 3-year prospective study. Clin Implant Dent Relat Res 2016;18(6):1119-1133. DOI: 10.1111/cid.12419.

27. Agliardi EL, Pozzi A, Stappert CF, et al. Immediate fixed rehabilitation of the edentulous maxilla: a prospective clinical and radiological study after 3 years of loading. Clin Implant Dent Relat Res 2014;16(2):292-302. DOI: 10.1111/j.1708-8208.2012.00482.x.

28. Crespi R, Vinci R, Capparé $P$, et al. A clinical study of edentulous patients rehabilitated according to the" all on four" immediate function protocol. Int J Oral Maxillofac Impl 2012;27(2):428-434.

29. Najafi $\mathrm{H}$, Siadat $\mathrm{H}$, Akbari S, et al. Effects of immediate and delayed loading on the outcomes of All-on-4 treatment: A prospective study. J Dentis (Tehran, Iran) 2016;13(6):415.
30. Hinze $M$, Thalmair $T$, Bolz $W$, et al. Immediate loading of fixed provisional prostheses using four implants for the rehabilitation of the edentulous arch: a prospective clinical study. Int J Oral Maxillofac Impl 2010;25(5):1011-1018.

31. Browaeys $\mathrm{H}$, Dierens $\mathrm{M}$, Ruyffelaert $\mathrm{C}$, et al. Ongoing crestal bone loss around implants subjected to computer-guided flapless surgery and immediate loading using the All-on- $4^{\circledR}$ concept. Clin Implant Dent Relat Res 2015;17(5):831-843. DOI: 10.1111/cid.12197.

32. Francetti $L$, Romeo D, Corbella $S$, et al. Bone level changes around axial and tilted implants in full-arch fixed immediate restorations. Interim results of a prospective study. Clin Implant Dent Relat Res 2012;14(5):646-654. DOI: 10.1111/j.1708-8208.2010. 00304.x.

33. Li S, Di P, Zhang Y, et al. Immediate implant and rehabilitation based on All-on-4 concept in patients with generalized aggressive periodontitis: A medium-term prospective study. Clin Implant Dent Relat Res 2017;19(3):559-571. DOI: 10.1111/cid.12483.

34. Agliardi EL, Francetti L, Romeo D, et al. Immediate rehabilitation of the edentulous maxilla: preliminary results of a single-cohort prospective study. Int J Oral Maxillofac Implants 2009;24(5):887-895.

35. Maló $\mathrm{P}$, de Araújo Nobre $\mathrm{M}$, Lopes $\mathrm{A}$, et al. Preliminary report on the outcome of tilted implants with longer lengths $(20-25 \mathrm{~mm})$ in low-density bone: one-year follow-up of a prospective cohort study. Clin Implant Dent Relat Res 2015;17:e134-e142. DOI: 10.1111/ cid.12144.

36. Piano $S$, Romeo $E$, Sbricoli $L$, et al. Simplified procedure for the immediate loading of a complete fixed prosthesis supported by four implants in the maxillary jaw: a 2-year prospective study. Clin Oral Implants Res 2016;27(12):e154-e160. DOI: 10.1111/ clr.12580.

37. Lopes A, Maló P, de Araújo Nobre M, et al. The NobelGuide ${ }^{\circledR}$ Allon $-4^{\oplus}$ treatment concept for rehabilitation of edentulous jaws: a retrospective report on the 7-years clinical and 5-years radiographic outcomes. Clin Implant Dent Relat Res 2017;19(2):233-244. DOI: 10.1111/cid.12456.

38. Maló P, de Araujo Nobre M, Petersson U, et al. A pilot study of complete edentulous rehabilitation with immediate function using a new implant design: case series. Clin Implant Dent Relat Res 2006;8(4):223-232. DOI: 10.1111/j.1708-8208.2006. 00024.x.

39. Sannino G, Barlattani A. Straight versus angulated abutments on tilted implants in immediate fixed rehabilitation of the edentulous mandible: A 3-year retrospective comparative study. Int J Prosthodont 2016;29(3):219-226. DOI: 10.11607/ijp.4448.

40. Acocella A, Ercoli C, Geminiani A, et al. Clinical evaluation of immediate loading of electroeroded screw-retained titanium fixed prostheses supported by tilted implant: A multicenter retrospective study. Clin Implant Dent Relat Res 2012;14:e98-e108. DOI: 10.1111/j.17088208.2011.00379.x.

41. Francetti L, Rodolfi A, Barbaro B, et al. Implant success rates in full-arch rehabilitations supported by upright and tilted implants: a retrospective investigation with up to five years of follow-up. J Periodon Implant Sci 2015;45(6):210-215. DOI: 10.5051/ jpis.2015.45.6.210.

42. Sannino G, Bollero P, Barlattani A, et al. A retrospective 2-year clinical study of immediate prosthetic rehabilitation of edentulous jaws with four implants and prefabricated bars. J Prosthodont 2017;26(5):387394. DOI: 10.1111/jopr.12406.

43. Mozzati M, Arata V, Gallesio G, et al. Immediate postextractive dental implant placement with immediate loading on four implants for mandibular-full-arch rehabilitation: a retrospective analysis. Clin Implant Dent Relat Res 2013;15(3):332-340. DOI: 10.1111/j.17088208.2011.00412.x.

44. Maló $P$, de Araújo Nobre $M$, Lopes $A$, et al. "All-on-4" immediatefunction concept for completely edentulous maxillae: a clinical report on the medium ( 3 years) and long-term ( 5 years) outcomes. 
Clin Implant Dent Relat Res 2012;14:e139-e150. DOI: 10.1111/j.17088208.2011.00395.x.

45. Malo P, de Araujo Nobre M, Lopes A. The use of computer-guided flapless implant surgery and four implants placed in immediate function to support a fixed denture: preliminary results after a mean follow-up period of thirteen months. J Prosthe Dentis 2007;97(6): S26-S34. DOI: 10.1016/S0022-3913(07)60005-5.

46. Tealdo T, Bevilacqua M, Pera F, et al. Immediate function with fixed implant-supported maxillary dentures: a 12-month pilot study. J Prosthe Dentis 2008;99(5):351-360. DOI: 10.1016/S00223913(08)60082-7.

47. Malo P, de Araújo Nobre M, Lopes A, et al. A longitudinal study of the survival of All-on-4 implants in the mandible with up to 10 years of follow-up. J Am Dent Associat 2011;142(3):310-320. DOI: 10.14219/ jada.archive.2011.0170.

48. Di P, Lin Y, Li JH, et al. The All-on-Four implant therapy protocol in the management of edentulous Chinese patients. Int J Prosthodont 2013;26(6):509-516. DOI: 10.11607/ijp.3602. 